\title{
Consensus Control of Multiagent Systems with High-Order Nonlinear Inaccurate Dynamics and Dynamically Switching Undirected Topologies
}

\author{
Qiang Wang $\mathbb{D}^{1},{ }^{1,2}$ Qingtian Meng, ${ }^{2}$ Xiaonan Fang, ${ }^{3}$ and Huaxiang Zhang ${ }^{1}$ \\ ${ }^{1}$ School of Information Science and Engineering, Shandong Normal University, Jinan 250014, China \\ ${ }^{2}$ School of Physics and Electronics, Shandong Normal University, Jinan 250014, China \\ ${ }^{3}$ School of Information Engineering, Shandong Management University, Jinan 250357, China \\ Correspondence should be addressed to Qiang Wang; wangqiang5169@163.com
}

Received 18 April 2018; Accepted 4 June 2018; Published 27 June 2018

Academic Editor: Xue-Jun Xie

Copyright ( 2018 Qiang Wang et al. This is an open access article distributed under the Creative Commons Attribution License, which permits unrestricted use, distribution, and reproduction in any medium, provided the original work is properly cited.

\begin{abstract}
This paper investigates the consensus control of a class of high-order nonlinear multiagent systems, whose topology is dynamically switching directed graph. First, the high-order nonlinear dynamics is transformed into the one-order dynamics by structuring a sliding mode plane; then, two consensus control protocols of the one-order dynamics are designed by feedback linearization, one of which is based on PD (proportion and derivative) and the other is based on PID (proportion, integral and derivative). Under these control protocols, it is proved that the consensus of new variable only requires a weaker topology condition; next, we prove that the consensus of the new variable is sufficient to the consensus of the states of multiagent systems, which implies that it only requires a weaker topology condition for the consensus of multiagent systems; finally, the study of an illustrative example with simulations shows that our results as well as designed control protocols work very well in studying the consensus of this class of multiagent systems.
\end{abstract}

\section{Introduction}

Nonlinear dynamics systems, inaccurate dynamics, and switching systems have been fascinating in the field of control and have attracted considerable attention worldwide. As is known to all, nonlinear dynamics [1-6] are more challenging than their linear peer. Inaccurate dynamics or noises increase the difficulty in analyzing the problem [7-10], and switching models, which some authors devote themselves to [11-15], are intractable. In this paper, we will focus on a class of special nonlinear and switching systems, that is, multiagent systems with nonlinear inaccurate dynamics and dynamically switching undirected topologies, and investigate its consensus conditions. As an important and fundamental issue of the multiagent systems, consensus problem is especially fascinating [16-19], and some specific scenarios on consensus problems are researched by scholars, such as time-delay [20-23], leader following consensus [24-27], and formation [28-31].
The consensus of multiagent systems with a switching interaction topology has been attracting many scholars [3234]. In [32], the authors considered multiagent systems with first-order integrator dynamics under the switching topology and provided a control protocol under which the dynamics could achieve consensus with a rather weak topology conditions. However, they considered the second-order integrator dynamics in [33] and obtained a condition that needs the stronger topology requirement than the one in [32]; what is more, they pointed out that the condition in [32] was not sufficient to the second-order consensus algorithm with their control protocol. The authors in [34] compared the control protocol in [32] with the one in [33] and pointed out that the topology requirement was dependent on the control protocol. They transformed the high-order linear systems to first-order integrator dynamics by variable substitution and obtained the consensus conditions of high-order linear multiagent systems with a rather weak topology requirement. 
The idea in [34] is interesting for high-order systems, which motivate us to consider a class of high-order nonlinear multiagent systems, whose topology is dynamically switching directed graph. We prove that the nonlinear dynamics can achieve consensus with a rather weak topology conditions similar to [32]. First, the high-order dynamics can realize dimensionality reduction by structuring a sliding mode plane; then, two consensus control protocols are designed by feedback linearization, one of which is based on PD (proportion and derivative), and the other is based on PID (proportion, integral, and derivative), such that the new model can achieve the consensus under a rather weak topology condition; next, we prove that the consensus of new control protocols is sufficient to the consensus of nonlinear multiagent systems. Thus, we give a rather weak consensus conditions of high-order nonlinear multiagent systems, in which the union graph has a spanning tree frequently enough. Finally, the study of an illustrative example with simulations shows that our results as well as designed control protocols work very well in studying this class of multiagent systems.

The main contribution of this paper contains: (1) a rather weak topology condition is given for the consensus of nonlinear multiagent systems with switching structure; (2) we present two control protocols for this class of systems, under which the consensus is proved; (3) the reduce-order idea is used to transform the high-order dynamics into one-order dynamics, which simplify the difficulty of problem.

The remainder of the paper is organized as follows. Section 2 is the backgrounds and preliminaries. Section 3 is the main results of the paper. In this section, the highorder nonlinear dynamics is transformed into one-order linear dynamics, and two control protocols are designed. In Section 4, we give an illustrative example to support our new results followed by the conclusion in Section 5 .

\section{Backgrounds and Preliminaries}

In [32], Ren and Beard considered a one-order linear system under the switching topology:

$$
x_{i}=u_{i}
$$

and they designed the control protocol as follows:

$$
u_{i}=-\sum_{j \in N_{i}(t)} a_{i j}\left(x_{i}-x_{j}\right)
$$

it was proved system (1) could achieve consensus under protocol (2) even if the switching topology satisfied a rather weak condition. Specifically to say, it could be formulated as the following lemma.

Lemma 1. Let $t_{1}, t_{2}, \ldots$ be an infinite time sequence at which the interaction graph or weighting factors $a_{i j}$ switch and $\tau_{i}=$ $t_{i+1}-t_{i} \in \gamma, i=0,1, \cdots$. Here, $\gamma$ is an infinite set generated from set $\widehat{\tau}$, which is a finite set of arbitrary positive numbers. Let $G\left(t_{i}\right)$ be a switching topology at time $t=t_{i}$ and all nonzero entries of the adjacency matrix $A=\left[a_{i j}\right]$ are lower bounded by a positive constant $\sigma_{L}$ and upper bounded by a positive constant $\sigma_{M}$. Then the distributed control protocols (2) achieve consensus asymptotically for the multiagent systems specified by (1) if there exists an infinite sequence of uniformly bounded, nonoverlapping time intervals $\left[t_{i_{j}}, t_{i_{j}+l_{j}}\right), l_{j} \in\left[1, i_{j+1}-i_{j}\right), j=$ $1,2 . \cdots$, starting at $t_{i_{1}}=t_{0}$, with the property that each interval $\left[t_{i_{j}+l_{j}}, t_{i_{j+1}}\right)$ is uniformly bounded and the union of the directed graphs across each interval $\left[t_{i_{j}+l_{j}}, t_{i_{j+1}}\right)$ has a spanning tree. Furthermore, the consensus value is a constant.

Furthermore, they considered the second-order integrator in [33]:

$$
\begin{aligned}
& \dot{x}_{i 1}=x_{i 2} \\
& \dot{x}_{i 2}=u_{i},
\end{aligned}
$$

where $x_{i}=\left[x_{i 1}, x_{i 2}\right]$ was the state of Agent $i$, and $u_{i}$ was the control protocol. They designed the control protocol as

$$
u_{i}=-\sum_{j=1}^{n} a_{i j}\left[\left(x_{i 1}-x_{j 1}\right)+r(t)\left(x_{i 2}-x_{j 2}\right)\right],
$$

where $r(t)$ was a positive scalar at time $t$. In that book, they provided a result which needed a more stronger connectivity assumption; that is, the interaction graph needed a directed spanning tree at each time instant. Moreover, they provided some simulation examples to illustrate that, under control protocol (4), the assumption of Lemma 1 could not ensure the consensus of second-order consensus generally.

$\mathrm{Su}$ and Lin compared the cases as above in [34] and presented a reduced-order idea. They considered the highorder linear multiagent dynamics as follows:

$$
\dot{\mathbf{x}}_{\mathbf{i}}=\mathbf{A} \mathbf{x}_{\mathbf{i}}+\mathbf{B} \mathbf{u}_{\mathbf{i}}, \quad i=1,2, \ldots, m,
$$

where $\mathbf{x}_{\mathbf{i}} \in R^{n}$ was the state vector of Agent $i, \mathbf{u}_{\mathbf{i}}$ was the control input the Agent $i$, and (A, B) was controllable. By transforming the system (5) to one-order dynamics, they presented a control protocol under which the high-order linear multiagent systems could obtain consensus with a rather weak topology.

Inspired by [34], we will consider a class of multiagent systems with $m$ agents, which each has $n$-order nonlinear dynamics described by

$$
x_{i}^{(n)}=f\left(x_{i}\right)+b\left(x_{i}\right) u_{i}, \quad i=1,2, \ldots, m,
$$

where $x_{i} \in \mathbf{R}$ is the state of agent $i, u_{i} \in \mathbf{R}$ is the control input of agent $i$, and $f\left(x_{i}\right)$ or $b\left(x_{i}\right)$ is inaccurate.

Denoting $x_{i 1}=x_{i}$, the dynamics (6) can be rewritten as

$$
\begin{gathered}
\dot{x}_{i 1}=x_{i 2}, \\
\dot{x}_{i 2}=x_{i 3} \\
\ldots, \\
\dot{x}_{i(n-1)}=x_{i n}, \\
\dot{x}_{i n}=f\left(x_{i}\right)+b\left(x_{i}\right) u_{i}, \quad i=1,2, \cdots, m,
\end{gathered}
$$

The objective of this study is to design a controller such that the agents described as the system (6) or (7) can 
achieve consensus under the dynamically switching directed interaction topologies.

In the following, we will provide some fundamental knowledge on algebraic graph theory, which will be used in the development of this research.

Suppose $\mathscr{G}=\{\mathscr{V}, \mathscr{E}\}$ be a directed graph of $N$-th order with the set of nodes $\mathscr{V}:=\left\{v_{1}, v_{2}, \cdots, v_{N}\right\}$, and the set of edges (i.e., ordered pairs of the agents) $\mathscr{E} \subseteq \mathscr{V} \times \mathscr{V}$. The matrix $\mathbf{A}=\left[a_{i j}\right]_{N \times N}$ is named the adjacency matrix of the graph $\mathscr{G}$, where $a_{i j}$ is the weight of Agent $i$ to Agent $j$. For any $i, j \in \mathscr{V}$, $a_{j i}>0$ if and only if $j \in \mathcal{N}_{i}$, where $\mathcal{N}_{i}=\left\{j \mid e_{i j}=\left(v_{i}, v_{j}\right) \in \mathscr{E}\right\}$. In this paper we just consider the case of simple graphs, that is, $e_{i i} \notin \mathscr{E}, i=1,2, \cdots, n$. The matrix $\mathbf{D}=\left[d_{i j}\right] \in \mathbf{R}^{N \times N}$ is the valency matrix of the topology $\mathscr{G}$, where $d_{i j}$ is defined as

$$
d_{i j}= \begin{cases}\sum_{k \in \mathscr{N}_{i}(t)} a_{i k}, & j=i, \\ 0, & j \neq i .\end{cases}
$$

Moreover, the matrix $\mathbf{L}=\mathbf{D}-\mathbf{A}$ is known as the graph's Laplacian matrix.

\section{Main Results}

This section studies the consensus of the multiagent dynamics (6). We will consider the following scenarios: (I) the dynamics $f\left(x_{i}\right), i=1,2, \cdots, m$ is inaccurate, but its estimate is known; (II) the gain $b\left(x_{i}\right), i=1,2, \cdots, m$ is inaccurate, but we know its upper bound and its lower bound.

Before giving our main results, another lemma will be useful in our study.

Lemma 2 (see [34]). Let $x(t)$ be a smooth function of $t$ and assume that $x(0), \dot{x}(0), \cdots, x^{(n-1)}(0)$ are all bounded. Define

$$
s(x, t)=\left(\lambda+\frac{d}{d t}\right)^{n-1} x(t),
$$

where $\lambda>0$ is a constant. If there exists a scalar $\Phi>0$ such that $|s(x(t), t)| \leq \Phi$ for all $t \geq 0$, then there exists a finite $T_{0}$, dependent on the values of $x(0), \dot{x}(0), \cdots, x^{(n-1)}(0)$, such that

$$
\left\|x^{(i)}(t)\right\| \leq \frac{2^{i} \Phi}{\lambda^{n-i-1}}, \quad t \geq T_{0}, i=0,1, \cdots, n-1 .
$$

3.1. Inaccurate Dynamics in the Systems. First, we consider the system (6) with undirected topology, assume that $\widehat{f}\left(x_{i}\right)$ is the estimate of $f\left(x_{i}\right)$, and the error of estimate is subject to $F\left(x_{i}, \dot{x}_{i}, \ddot{x}_{i}, \cdots, x_{i}^{(n-1)}\right)$, that is, $\left|f\left(x_{i}\right)-\widehat{f}\left(x_{i}\right)\right| \leq F$.

Denote

$$
z_{i}(t)=\left(\lambda+\frac{d}{d t}\right)^{n-1} x_{i}(t), \quad i=1,2, \cdots, N
$$

where $\lambda$ is a positive number.

Then design the control protocol as

$$
\begin{aligned}
\widehat{u}_{i}= & -\sum_{j=1}^{m} a_{i j}(t)\left(z_{i}-z_{j}\right)-C_{n-1}^{0} \lambda^{n-1} x_{i 2}-C_{n-1}^{1} \lambda^{n-2} x_{i 3} \\
& -C_{n-1}^{2} \lambda^{n-3} x_{i 4}-\ldots-C_{n-1}^{n-2} \lambda x_{i n}-\widehat{f}\left(x_{i}\right),
\end{aligned}
$$

and

$$
u_{i}=\widehat{u}_{i}-F \operatorname{sgn}\left(z_{i}\right)
$$

where

$$
\operatorname{sgn}\left(z_{i}\right)= \begin{cases}1, & z_{i} \geq 0, \\ 0, & z_{i}<0,\end{cases}
$$

under the protocol (13), and we have the following result.

Theorem 3. Consider the multiagent system of $m$ agents (6) under the control protocol specified by (13). If the conditions of Lemma 1 are satisfied, then the distributed control protocol (13) achieves consensus asymptotically for the multiagent systems specified by (6) and the consensus is reached at a constant state $[c, 0,0, \cdots, 0]^{T}$.

Proof. Take derivative for (11), and we have

$$
\begin{aligned}
& \dot{z}_{i}(t)=\frac{d}{d t}\left\{\left(\lambda+\frac{d}{d t}\right)^{n-1} x_{i 1}\right\}=\frac{d}{d t}\left\{C_{n-1}^{0} \lambda^{n-1} x_{i 1}\right. \\
& +C_{n-1}^{1} \lambda^{n-2} x_{i 2}+C_{n-1}^{2} \lambda^{n-3} x_{i 3}+\ldots+C_{n-1}^{n-2} \lambda x_{i(n-1)} \\
& \left.+x_{i n}\right\}=C_{n-1}^{0} \lambda^{n-1} x_{i 2}+C_{n-1}^{1} \lambda^{n-2} x_{i 3}+C_{n-1}^{2} \lambda^{n-3} x_{i 4} \\
& +\ldots+C_{n-1}^{n-2} \lambda x_{i n}+f\left(x_{i}\right)+u_{i} \\
& =-\sum_{j \in N_{i}(t)} a_{i j}\left(z_{i}-z_{j}\right)+f-\widehat{f}-F \operatorname{sgn}\left(z_{i}\right),
\end{aligned}
$$

Choose a Lyapunov function candidate

$$
V(z)=\frac{1}{2} z^{T} z
$$

where $z=\left[z_{1}, z_{2}, \cdots, z_{m}\right]^{T}$; then

$$
\begin{aligned}
\dot{V}(z) & =\dot{z}^{T} z \\
& =-\sum_{i=1}^{m}\left(\sum_{j=1}^{m} a_{i j}\left(z_{i}-z_{j}\right) z_{j}+(f-\widehat{f}) z_{i}-F\left|z_{i}\right|\right) \\
& \leq-\sum_{i=1}^{m}\left(\sum_{j=1}^{m} a_{i j}\left(z_{i}-z_{j}\right) z_{j}+|f-\widehat{f}|\left|z_{i}\right|-F\left|z_{i}\right|\right) \\
& =-\sum_{i=1}^{m}\left(\sum_{j=1}^{m} a_{i j}\left(z_{i}-z_{j}\right) z_{j}+(|f-\widehat{f}|-F)\left|z_{i}\right|\right) \\
& \leq-\sum_{j=1}^{m} \sum_{i=1}^{m} a_{i j}\left(z_{i}-z_{j}\right) z_{j}=-\frac{1}{2} \sum_{i=1}^{m} \sum_{j=1}^{m} a_{i j}\left(z_{i}-z_{j}\right)^{2} \\
& \leq 0,
\end{aligned}
$$

due to $|f-\widehat{f}| \leq F$.

Letting $\dot{V}(z)=0$, that is, $\sum_{i=1}^{m} \sum_{j=1}^{m} a_{i j}\left(z_{i}-z_{j}\right)^{2}=0$, we have $z_{i}=z_{j}, i, j=1,2, \ldots, m$. According to LaSalle invariant set, the systems converge to the state $z_{i}=z_{j}, i=1,2, \cdots, m$. 
On the other hand, (11) implies $z_{i}(t)-z_{j}(t)=(\lambda+$ $d / d t)^{n-1}\left(x_{i 1}(t)-x_{j 1}(t)\right)$. Since $z_{i}, z_{j}, i, j=1,2, \cdots, m$ achieves consensus asymptotically, then $\forall \epsilon>0, \exists T_{1}>0$, such that when $t>T_{1},\left|z_{i}(t)-z_{j}(t)\right|<\epsilon$. According to Lemma 2, there exists a finite $T_{0}>T_{1}$, dependent on the values of $x_{i}\left(T_{1}\right), \dot{x}_{i}\left(T_{1}\right), \cdots, x_{i}^{(n-1)}\left(T_{1}\right), i=1,2, \cdots, N$, such that

$$
\begin{aligned}
\left\|x_{i 1}^{(k)}(t)-x_{j 1}^{(k)}(t)\right\| \leq 2^{k} \epsilon / \lambda^{n-k-1}, & \\
& t \geq T_{0}, k=0,1, \cdots, n-1,
\end{aligned}
$$

which implies that

$$
\left\|x_{i s}(t)-x_{j s}(t)\right\| \leq \epsilon_{0}, \quad t \geq T_{0}, s=1,2, \cdots, n,
$$

where $\epsilon_{0}=\max \left\{2^{k} \epsilon / \lambda^{n-k-1}, k=0,1, \cdots, n-1\right\}$ is an arbitrarily small positive number, which implies that $x_{i}(t)$ achieves consensus asymptotically.

Consider the dynamics (7), since $x_{i}(t)$ achieves consensus asymptotically, it is easy to obtain that

$$
\lim _{t \rightarrow+\infty} \dot{x}_{i k}=0, \quad k=1,2, \ldots, n,
$$

which implies that $x_{i 1} \rightarrow c$ and $x_{i k} \rightarrow 0, k=2,3, \cdots, n$, thus, $x_{i} \rightarrow[c, 0,0, \cdots, 0]^{T}$.

However, the terms $F \operatorname{sgn}\left(z_{i}\right)$ have a strong flutter, which may need a strong control power. Next, we will consider the stable under any precision.

Definition 4. The system $\dot{x}=f(x)$ is said to any precision stable if for any designated $\epsilon_{0}$, there exists $T$, such that $\|x(t)\|<\epsilon_{0}$, when $t>T$.

Consider the set $B(t)=\{x \mid s(x, t) \leq \Phi\}$, where $\Phi>0$ is the precision of system state. Letting

$$
u_{i}=\widehat{u}_{i}-F \operatorname{sat}\left(\frac{z_{i}}{\Phi}\right),
$$

where $u_{i}$ is the same as (12),

$$
\operatorname{sat}\left(\frac{z_{i}}{\Phi}\right)= \begin{cases}\frac{z_{i}}{\Phi}, & \left|z_{i}\right| \leq \Phi, \\ \operatorname{sgn}\left(z_{i}\right), & \left|z_{i}\right|>\Phi,\end{cases}
$$

under the control protocol (21), we have the following theorem.

Theorem 5. Consider the multiagent system of $m$ agents (6), which is steered by the control protocol specified by (21). If the conditions of Lemma 1 are satisfied, then the protocol (21) achieves any precision stable for the multiagent systems specified by (6).

Proof. When $\left|z_{i}\right| / \Phi>0$,

$$
u_{i}=\widehat{u}_{i}-F \operatorname{sgn}\left(z_{i}\right),
$$

from the proof of Theorem 3 , the dynamics are convergent to the invariant set $B(t)$; that is, there exists a constant $T_{0}$, when $t>T_{0}, z_{i}\left(x_{i}, t\right) \leq \Phi$. According to Lemma 2 ,

$$
\left\|x^{(i)}(t)\right\| \leq \frac{2^{i} \Phi}{\lambda^{n-i-1}}, \quad t \geq T_{0}, \quad i=0,1, \cdots, n-1 .
$$

Thus, $x_{i}(t) \leq \Phi / \lambda^{n-1}$ for $i=1,2, \ldots, m$.

3.2. Inaccurate Gain in the Systems. Consider system (6) with the inaccurate gain, that is, $b(x)$ is unknown, but we know its bounds, $\underline{b} \leq b\left(x_{i}\right) \leq \bar{b}, i=1,2, \ldots, N$, where $\underline{b}$ and $\bar{b}$ are positive constant number.

Denoting $\widehat{b}=\sqrt{\underline{b} \bar{b}}$, and designing

$$
\begin{aligned}
u_{i}= & -\widehat{b}^{-1} \sum_{j=1}^{N}\left(s_{i}-s_{j}\right)-C_{n-1}^{0} \lambda^{n-1} x_{i 2}-C_{n-1}^{1} \lambda^{n-2} x_{i 3} \\
& -C_{n-1}^{2} \lambda^{n-3} x_{i 4}-\ldots-C_{n-1}^{n-2} \lambda x_{i n}-f\left(x_{i}\right),
\end{aligned}
$$

then system (6) can be rewritten as

$$
\dot{s}_{i}=-\sum_{j=1}^{N} b\left(x_{i}\right) \widehat{b}^{-1} a_{i j}\left(s_{i}-s_{j}\right)
$$

Letting $\widehat{\beta}=\sqrt{\bar{b} / \underline{b}}$, then $\widehat{\beta}>1$. Since $\underline{b} \leq b\left(x_{i}\right) \leq \bar{b}$, then $\widehat{\beta}^{-1} \leq b\left(x_{i}\right) \widehat{b}^{-1} \leq \widehat{\beta}$

The control protocol (12) with (11) is constructed by PD Control, which can bring in steady-state error. Thus, another control protocol is designed in order to overcome this deficiency.

Letting

$$
z_{i}(t)=\left(\lambda+\frac{d}{d t}\right)^{n} \int_{0}^{t} x_{i}(r) d r, \quad i=1,2, \cdots, N,
$$

the control protocol is designed as follows:

$$
\begin{aligned}
u_{i}= & -\sum_{j \in N_{i}(t)} a_{i j}\left(z_{i}-z_{j}\right)-C_{n}^{0} \lambda^{n} x_{i 1}-C_{n}^{1} \lambda^{n-1} x_{i 2} \\
& -C_{n}^{2} \lambda^{n-2} x_{i 3}-\ldots-C_{n}^{n-1} \lambda x_{i n}-f\left(x_{i}\right),
\end{aligned}
$$

and we have the following conclusion.

Theorem 6. Consider the multiagent system of $m$ agents (6), which is steered by the control protocol specified by (28). If and the consensus is reached at a constant state $[c, 0,0, \cdots, 0]^{T}$. If the conditions of Lemma 1 are satisfied, then the distributed control protocol (28) achieves consensus asymptotically for the multiagent systems specified by (6). 
Proof. Since

$$
\begin{aligned}
\dot{z}_{i}( & t)=\frac{d}{d t}\left\{\left(\lambda+\frac{d}{d t}\right)^{n} \int_{0}^{t} x_{i}(r) d r\right\} \\
= & \frac{d}{d t}\left\{C_{n}^{0} \lambda^{n} \int_{0}^{t} x_{i}(r) d r+C_{n}^{1} \lambda^{n-1} x_{i 1}+C_{n}^{2} \lambda^{n-2} x_{i 2}\right. \\
& \left.+\ldots+C_{n}^{n-1} \lambda x_{i(n-1)}+C_{n}^{n} x_{i n}\right\}=C_{n}^{0} \lambda^{n} x_{i 1} \\
& +C_{n}^{1} \lambda^{n-1} x_{i 2}+C_{n}^{2} \lambda^{n-2} x_{i 3}+\ldots+C_{n}^{n-1} \lambda x_{i n}+\dot{x}_{i n} \\
& =C_{n}^{0} \lambda^{n} x_{i 1}+C_{n}^{1} \lambda^{n-1} x_{i 2}+C_{n}^{2} \lambda^{n-2} x_{i 3}+\ldots \\
& +C_{n}^{n-1} \lambda x_{i n}+f\left(x_{i}\right)+u_{i}=-\sum_{j \in N_{i}(t)} a_{i j}\left(z_{i}-z_{j}\right)
\end{aligned}
$$

according to Lemma 1, the dynamics (29) can achieve consensus asymptotically if there exist infinite sequence of uniformly bounded, nonoverlapping time intervals $\left[t_{i_{j}}, t_{i_{j}+l_{j}}\right), l_{j} \in\left[1, i_{j+1}-i_{j}\right), j=1,2, \cdots$ starting at $t_{i_{1}}=t_{0}$, with the property that each interval $\left[t_{i_{j}+l_{j}}, t_{i_{j+1}}\right)$ is uniformly bounded and the union of the directed graphs across each interval $\left[t_{i_{j}+l_{j}}, t_{i_{j+1}}\right)$ has a spanning tree.

On the other hand, (27) implies $z_{i}(t)-z_{j}(t)=(\lambda+$ $d / d t)^{n}\left(\int_{0}^{t}\left(x_{i 1}(r)-x_{j 1}(r)\right) d r\right)$. Since the dynamics (29) achieve consensus asymptotically, then $\forall \epsilon>0, \exists T_{1}>0$, such that when $t>T_{1},\left|z_{i}(t)-z_{j}(t)\right|<\epsilon$. According to Lemma 2, there exists a finite $T_{0}>T_{1}$, dependent on the values of $\int_{0}^{T_{1}} x_{i}(r) d r, x_{i}\left(T_{1}\right), \dot{x}_{i}\left(T_{1}\right), \cdots, x_{i}^{(n-1)}\left(T_{1}\right), i=1,2, \cdots, N$, such that

$$
\left\|\int_{0}^{t}\left(x_{i 1}(r)-x_{j 1}(r)\right) d r\right\| \leq \frac{\epsilon}{\lambda^{n}}, \quad t \geq T_{0}
$$

and

$$
\begin{aligned}
&\left\|x_{i 1}^{(k)}(t)-x_{j 1}^{(k)}(t)\right\| \leq \frac{2^{k} \epsilon}{\lambda^{n-k-1}}, \\
& \quad t \geq T_{0}, k=0,1, \cdots, n-1 .
\end{aligned}
$$

According to the proof of Theorem 3, (31) implies

$$
\left\|x_{i s}(t)-x_{j s}(t)\right\| \leq \epsilon_{0}, \quad t \geq T_{0}, s=1,2, \cdots, n .
$$

where $\epsilon_{0}=\max \left\{2^{k} \epsilon / \lambda^{n-k-1}, k=0,1, \cdots, n-1\right\}$ is an arbitrarily small positive number, which implies that $x_{i}(t)$ achieves consensus asymptotically.

Consider the dynamics (7); since $x_{i}(t)$ achieves consensus asymptotically, it is easy to obtain that

$$
\lim _{t \rightarrow+\infty} \dot{x}_{i k}=0, \quad k=1,2, \ldots, n,
$$

which implies that $x_{i 1} \rightarrow c$ and $x_{i k} \rightarrow 0, k=2,3, \cdots, n$, thus, $x_{i} \rightarrow[c, 0,0, \cdots, 0]^{T}$.

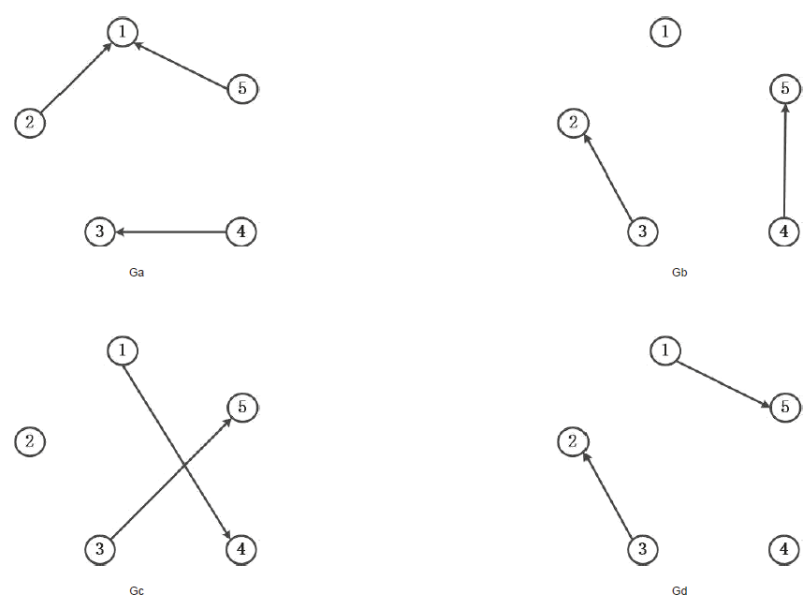

FIGURE 1: The topology of switching directed graph.

Remark 7. The method provided in this research is suitable for heterogeneous multiagent systems:

$$
x_{i}^{(n)}=f_{i}\left(x_{i}\right)+u_{i}, \quad i=1,2, \ldots, m,
$$

only if the control protocol is designed as

$$
\begin{aligned}
u_{i}= & -\sum_{j \in N_{i}(t)} a_{i j}\left(z_{i}-z_{j}\right)-C_{n}^{0} \lambda^{n} x_{i 1}-C_{n}^{1} \lambda^{n-1} x_{i 2} \\
& -C_{n}^{2} \lambda^{n-2} x_{i 3}-\ldots-C_{n}^{n-1} \lambda x_{i n}-f_{i}\left(x_{i}\right) .
\end{aligned}
$$

\section{Illustrative Examples}

In this section, we provide an illustrative example to show how to use the method in this research to design control protocol for the consensus of this class of multiagent systems.

Example 1. Consider the following 5-agent system running on a circle:

$$
\begin{aligned}
& \dot{x}_{i 1}=x_{i 2}, \\
& \dot{x}_{i 2}=x_{i 3}, \\
& x_{i 3}=\sum_{j=1}^{3} x_{i j}^{2}+u_{i}, \quad i=1,2,3,4,5,
\end{aligned}
$$

whose topology is shown as Figure 1, $\{G a, G b, G c, G d\}$ is the set of switching directed graph, it is easy to see that each of them does not obtain a spanning tree. To show the correctness of the above conclusion, we carry out the following numerical simulations. The dynamics start at $\mathrm{Ga}$ and switch to the next one after $T=0.01$, and the switching rules of network topology are as follows: $G a \rightarrow G b \rightarrow G c \rightarrow$ $G d \rightarrow G a \rightarrow G b \cdots$. It is easy to see that the union graph of the dynamics system (36) has a spanning tree frequently enough. 

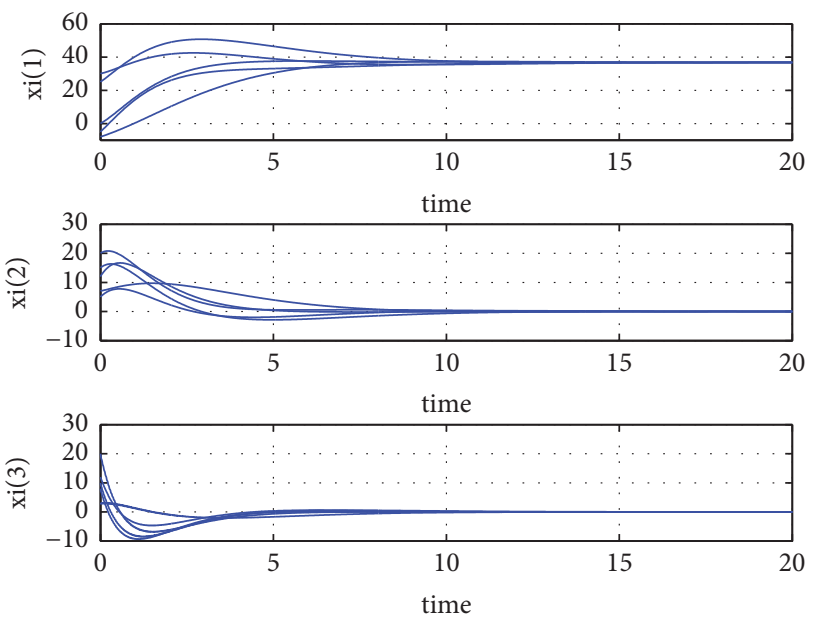

Figure 2: The states of agents under the control protocol (37).

Design the control protocol

$$
\begin{array}{r}
u_{i}=-\sum_{j \in N_{i}(t)}\left(z_{i}-z_{j}\right)-C_{2}^{0} \lambda^{2} x_{i 2}-C_{2}^{1} \lambda x_{i 3}-\sum_{j=1}^{3} x_{i j}^{2}, \\
i=1,2,3,4,5,
\end{array}
$$

where $z_{i}(t)=(\lambda+d / d t)^{2} x_{i}(t)$. Herein, let $\lambda=1$ and initial condition $x_{1}(0)=[25,15,10]^{T}, x_{2}(0)=[30,5,12]^{T}, x_{3}(0)=$ $[-5,20,8]^{T}, x_{4}(0)=[-8,7,3]^{T}, x_{5}(0)=[0,12,20]^{T}$.

The simulation results are shown in Figure 2, from which we can see that the states of the 5 agents eventually converge to the same value under the protocol (37) and the final result coincides with the theoretical analysis. Simulation shows that our method is very effective in analyzing the consensus of this kind of multiagent system (36).

In the following, we consider Example 1 with the same switching topology and the same switching rules and design a control protocol obtaining the integration as follows:

$$
\begin{aligned}
u_{i}= & -\sum_{j \in N_{i}(t)}\left(z_{i}-z_{j}\right)-C_{3}^{0} \lambda^{3} \int_{0}^{t} x_{i 1}(r) d r-C_{3}^{1} \lambda^{2} x_{i 1} \\
& -C_{3}^{2} \lambda x_{i 2}-C_{3}^{2} x_{i 3}-\sum_{j=1}^{3} x_{i j}^{2},
\end{aligned}
$$

Under the same initial conditions and $\lambda=1$, the simulation results are shown in Figure 3, from which we can see that the agents converge to each other under the control protocol (38).

\section{Conclusion}

In this paper, the consensus control of a class of high-order nonlinear multiagent systems was investigated, whose topology switched dynamically, and we obtained some consensus results on this class of multiagent systems. Herein, a rather weak topology condition was given for the consensus of nonlinear multiagent systems with switching structure, and
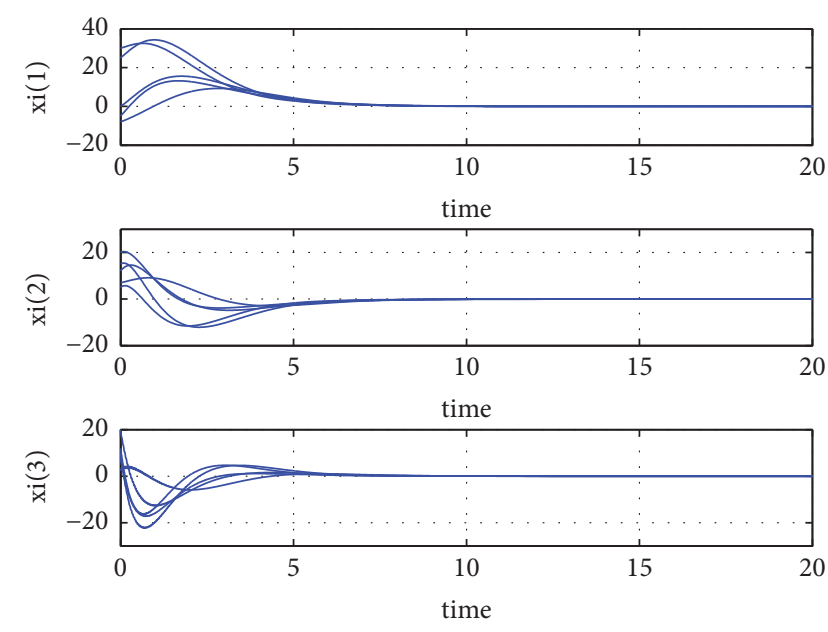

FIgURE 3: The states of agents under the control protocol (38).

two control protocols, one of which was based on PD and the other was based on PID, were presented for this class of systems, and the stabilization was proved; furthermore, a reduced-order method was used to handle the high-order dynamics system, which simplify the difficulty of problem. Simulations showed that our results as well as designed control protocols worked very well in studying the consensus of this class of multiagent systems. However, we just take a step for this class of systems, and some questions, such as how to obtain the estimate $\widehat{f}(x)$, will be considered in our subsequent research.

\section{Data Availability}

The data used to support the findings of this study are included within the article (Figures 2 and 3 ).

\section{Conflicts of Interest}

The authors declare that they have no conflicts of interest.

\section{Acknowledgments}

This paper was supported by the National Nature Science Foundation of China [61572298, 61602283, 61702310] and the Natural Science Foundation of Shandong, China (no. ZR2016FB10).

\section{References}

[1] W. Sun, K. Wang, C. Nie, and X. Xie, "Energy-based controller design of stochastic magnetic levitation system," Mathematical Problems in Engineering, Art. ID 7838431, 6 pages, 2017.

[2] L. Wang and Q.-G. Wang, "A general approach for synchronisation of nonlinear networked systems with switching topology," International Journal of Systems Science, vol. 44, no. 12, pp. 21992210, 2013.

[3] W. Sun, G. Pang, P. Wang, and L. Peng, "Robust adaptive control and L2 disturbance attenuation for uncertain Hamiltonian systems with time-delay," Mathematical Problems in Engineering, Art. ID 183279, 10 pages, 2013. 
[4] W. Sun and L. Peng, "Observer-based robust adaptive control for uncertain stochastic Hamiltonian systems with state and input delays," Nonlinear Analysis: Modelling and Control, vol. 19, no. 4, pp. 626-645, 2014.

[5] W. Sun and B. Fu, "Adapative control of time-varying uncertain non-linear systems with input delay: a Hamiltonian approach," IET Control Theory \& Applications, vol. 10, no. 15, pp. 1844-1858, 2016.

[6] W. Sun and L. Peng, "Robust adaptive control of uncertain stochastic Hamiltonian systems with time varying delay," Asian Journal of Control, vol. 18, no. 2, pp. 642-651, 2016.

[7] X. Song, J. H. Park, and X. Yan, "Linear estimation for measurement-delay systems with periodic coefficients and multiplicative noise," Institute of Electrical and Electronics Engineers Transactions on Automatic Control, vol. 62, no. 8, pp. 4124-4130, 2017.

[8] X. Song and J. H. Park, "Linear optimal estimation for discretetime measurement delay systems with multichannel multiplicative noise," IEEE Transactions on Circuits and Systems II: Express Briefs, vol. 64, no. 2, pp. 156-160, 2017.

[9] X. Song and J. H. Park, "Linear minimum mean-square estimation for discrete-time measurement-delay systems with multiplicative noise and Markov jump," IET Control Theory \& Applications, vol. 10, no. 10, pp. 1161-1169, 2016.

[10] X. Song and X. Yan, "Duality of linear estimation for multiplicative noise systems with measurement delay," IET Signal Processing, vol. 7, no. 4, pp. 277-284, 2013.

[11] X. Liu, K. Zhang, S. Li, S. Fei, and H. Wei, "Optimal control of switching times in switched stochastic systems," Asian Journal of Control, vol. 17, no. 5, pp. 1580-1589, 2015.

[12] X. Liu, K. Zhang, S. Li, S. Fei, and H. Wei, "Optimal timing control of switched stochastic systems," IMA Journal of Mathematical Control and Information, vol. 32, no. 3, pp. 659-674, 2015.

[13] X. Liu, S. Li, and K. Zhang, "Optimal control of switching time in switched stochastic systems with multi-switching times and different costs," International Journal of Control, vol. 90, no. 8, pp. 1604-1611, 2017.

[14] Y. Zhang and Y.-P. Tian, "Consentability and protocol design of multi-agent systems with stochastic switching topology," Automatica, vol. 45, no. 5, pp. 1195-1201, 2009.

[15] Y. Hong, L. Gao, D. Cheng, and J. Hu, "Lyapunov-based approach to multiagent systems with switching jointly connected interconnection," Institute of Electrical and Electronics Engineers Transactions on Automatic Control, vol. 52, no. 5, pp. 943-948, 2007.

[16] F. Zhang, W. Wang, and H. Zhang, "Distributed design of approximately optimal controller for identical discrete-time multi-agent systems," Journal of Systems Science \& Complexity, vol. 29, no. 3, pp. 629-641, 2016.

[17] F. Zhang, H. Zhang, C. Tan, W. Wang, and J. Gao, "A new approach to distributed control for multi-agent systems based on approximate upper and lower bounds," International Journal of Control, Automation, and Systems, vol. 15, no. 6, pp. 25072515, 2017.

[18] G. Z. Cui, S. Y. Xu, Q. Ma, Y. M. Li, and Z. Q. Zhang, "Prescribed performance distributed consensus control for nonlinear multiagent systems with unknown dead-zone input," International Journal of Control, vol. 91, no. 5, 2018.

[19] H. Liu, P. Zhang, B. Hu, and P. Moore, "A novel approach to task assignment in a cooperative multi-agent design system," Applied Intelligence, vol. 43, no. 1, pp. 162-175, 2015.
[20] Z. Wang, H. Zhang, X. Song, and H. Zhang, "Consensus problems for discrete-time agents with communication delay," International Journal of Control, Automation, and Systems, vol. 15, no. 4, pp. 1515-1523, 2017.

[21] Z. Wang, J. Xu, X. Song, and H. Zhang, "Consensus Conditions for Multi-agent Systems Under Delayed Information," IEEE Transactions on Circuits and Systems II: Express Briefs, pp. 1-1.

[22] R. Olfati-Saber and R. M. Murray, "Consensus problems in networks of agents with switching topology and time-delays," IEEE Transactions on Automatic Control, vol. 49, no. 9, pp. 15201533, 2004.

[23] H.-Y. Yang, G.-D. Zong, and S.-Y. Zhang, "Movement consensus of delayed multi-agent systems with directed weighted networks," International Journal of Intelligent Computing and Cybernetics, vol. 4, no. 2, pp. 265-277, 2011.

[24] H. Michael, Z. Chen, X. Wang, and J. Lam, "Semi-global observer-based leader-following consensus with input saturation," IEEE Transactions on Industrial Electronics, vol. 61, no. 6, pp. 2842-2850, 2014.

[25] B. Liu, T. Chu, L. Wang, and G. Xie, "Controllability of a leaderfollower dynamic network with switching topology," Institute of Electrical and Electronics Engineers Transactions on Automatic Control, vol. 53, no. 4, pp. 1009-1013, 2008.

[26] P. Li, S. Xu, Q. Ma, W. Chen, and Z. Zhang, "Leader-following rendezvous for uncertain Euler-Lagrange multi-agent systems by output feedback," Journal of The Franklin Institute, vol. 354, no. 10, pp. 4215-4230, 2017.

[27] C. Ma, T. Li, and J. Zhang, "Consensus control for leader-following multi-agent systems with measurement noises," Journal of Systems Science \& Complexity, vol. 23, no. 1, pp. 35-49, 2010.

[28] Q. Wang, Y. Wang, and H. Zhang, "The formation control of multi-agent systems on a circle," IEEE/CAA Journal of Automatica Sinica, vol. 5, no. 1, pp. 148-154, 2018.

[29] C. Q. Ma and J. F. Zhang, "On formability of linear continuous multi-agent systems," Journal of Systems Science and Complexity, vol. 25, no. 1, pp. 13-29, 2012.

[30] Y. Sun, Y. Tian, and X.-J. Xie, "Stabilization of positive switched linear systems and its application in consensus of multiagent systems," Institute of Electrical and Electronics Engineers Transactions on Automatic Control, vol. 62, no. 12, pp. 6608-6613, 2017.

[31] J. Wang, B. Gong, H. Liu, and S. Li, "Multidisciplinary approaches to artificial swarm intelligence for heterogeneous computing and cloud scheduling," Applied Intelligence, vol. 43, no. 3, pp. 662-675, 2015.

[32] W. Ren and R. W. Beard, "Consensus seeking in multiagent systems under dynamically changing interaction topologies," IEEE Transactions on Automatic Control, vol. 50, no. 5, pp. 655661, 2005.

[33] W. Ren and R. W. Beard, Distributed consensus in multi-vehicle cooperative control: theory and applications, Springer, London, U.K, 2008.

[34] S. Su and Z. Lin, "Distributed Consensus Control of MultiAgent Systems with Higher Order Agent Dynamics and Dynamically Changing Directed Interaction Topologies," IEEE Transactions on Automatic Control, vol. 61, no. 2, pp. 515-519, 2016. 


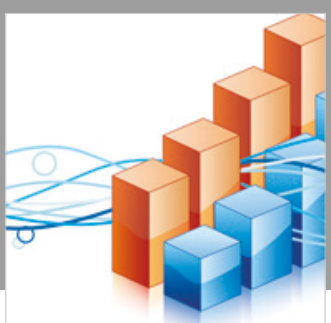

Advances in

Operations Research

\section{-n-m}
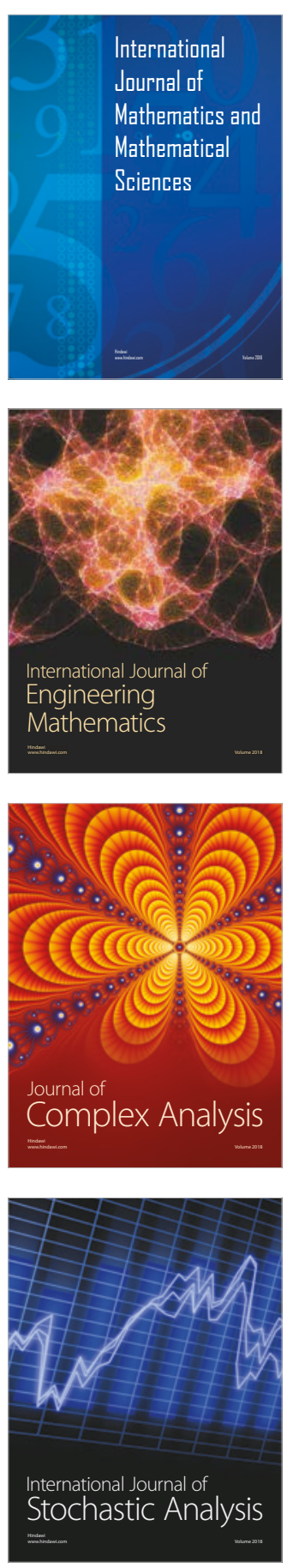
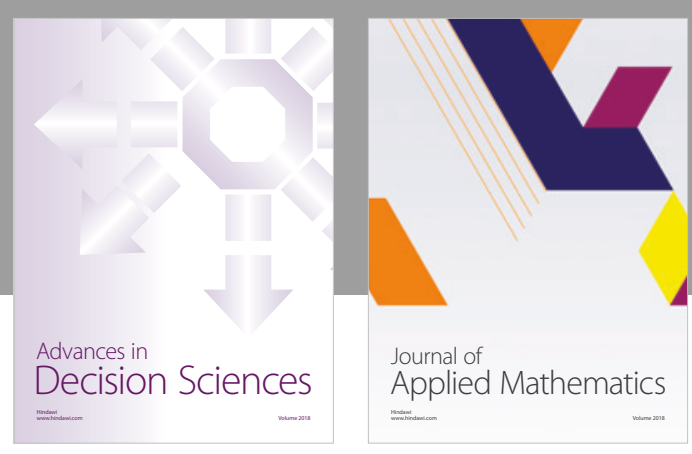

Journal of

Applied Mathematics
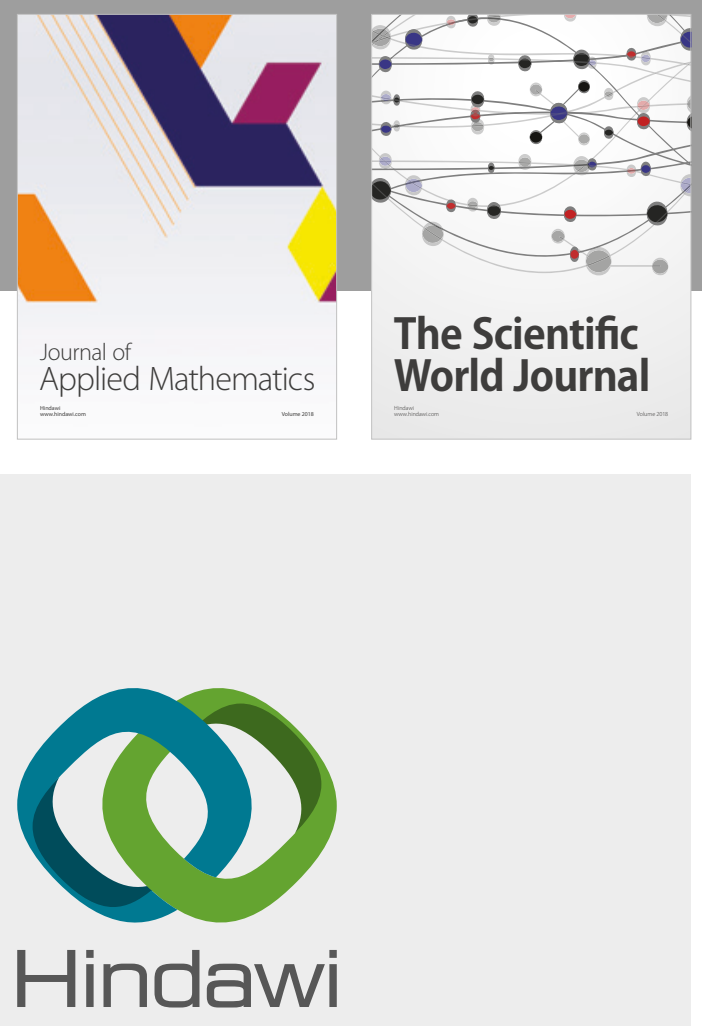

Submit your manuscripts at

www.hindawi.com

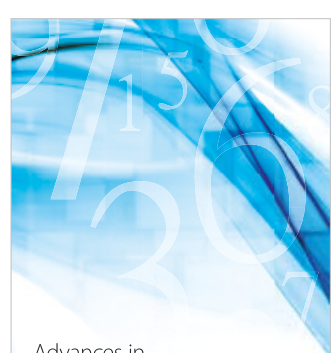

Advances in
Numerical Analysis
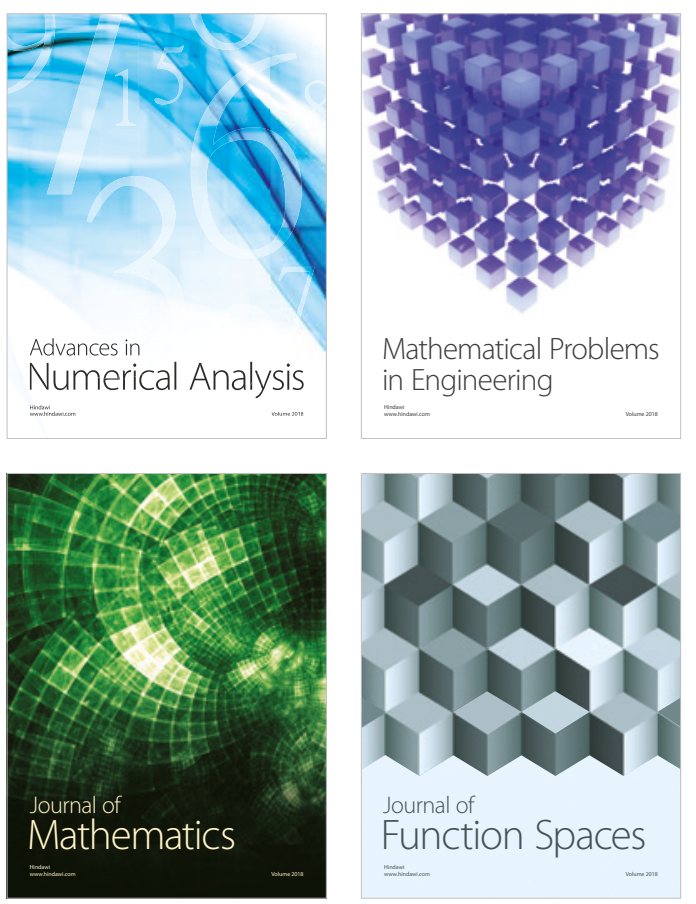

Mathematical Problems in Engineering

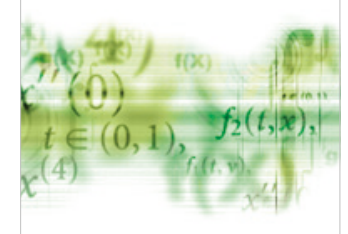

International Journal of

Differential Equations

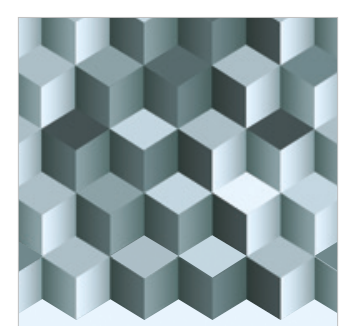

Journal of

Function Spaces

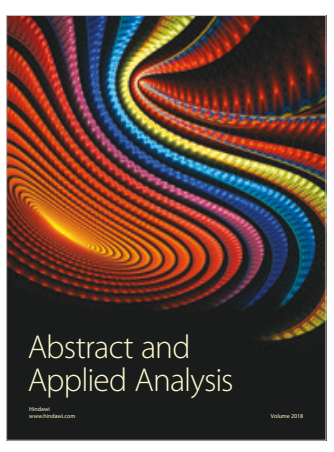

The Scientific

World Journal

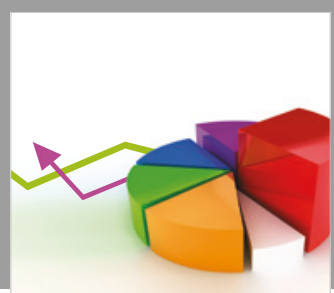

Journal of

Probability and Statistics
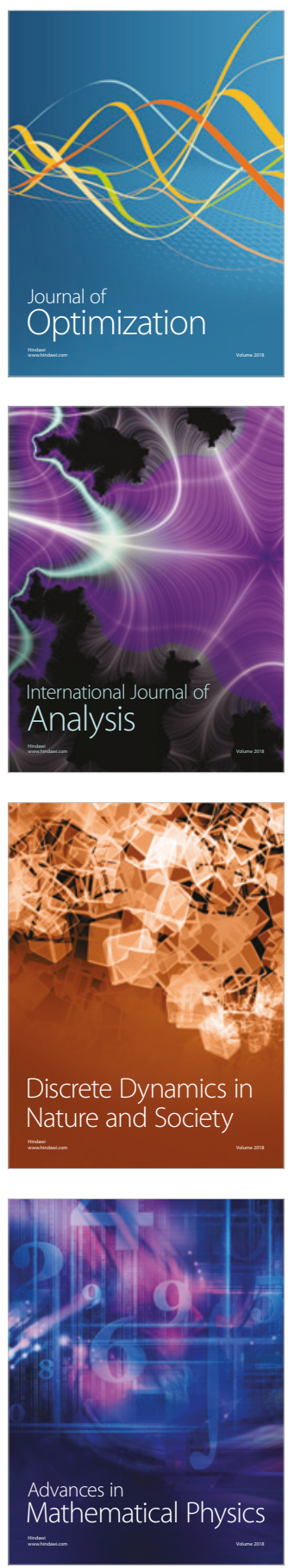